\title{
COMPARAÇÃO DE MÉTODOS DE AMOSTRAGEM PARA ANÁLISE ESTRUTURAL DE FLORESTAS INEQÜIÂNEAS ${ }^{1}$
}

\author{
Cássia Aparecida de Farias², Carlos Pedro Boëchat Soares ${ }^{3}$, Agostinho Lopes de Souza $^{3}$ e Helio Garcia Leite ${ }^{3}$
}

\begin{abstract}
RESUMO - O presente trabalho foi realizado em um povoamento florestal ineqüiâneo, pertencente à Universidade Federal de Viçosa-MG, objetivando comparar estimativas do número de árvores por hectare, volume por hectare, área basal por hectare, diâmetro médio e altura média do povoamento e número de espécies, utilizando parcelas de área fixa (método I) e amostragem por ponto horizontal (método de Bitterlich), com fatores de área basal $\mathrm{K}=1$, $\mathrm{K}=2$ e $\mathrm{K}=4$ (métodos II, III e IV, respectivamente). Após análises, constatou-se que: a) para atender a um determinado erro de amostragem, há a necessidade de maior número de pontos de amostragem, em comparação ao número de parcelas de área fixa; b) não houve diferença estatística entre as estimativas de volume por hectare, área basal por hectare, diâmetro médio e altura média do povoamento, obtidas nas parcelas de área fixa e nos pontos de amostragem, independentemente do fator de área basal; c) a amostragem por ponto horizontal (método de Bitterlich) poderá ser utilizada para caracterização da composição florística se houver aumento de intensidade amostral ou se a floresta apresentar baixa diversidade de espécies; e d) houve diferença estatística entre o número de árvores por hectare e por classe de diâmetro para os métodos de amostragem estudados. Os métodos II, III e IV subestimaram o número de árvores nas maiores classes de dap (diâmetro à altura do peito) e superestimaram-no nas menores.
\end{abstract}

Palavras-chave: Floresta ineqüiânea, métodos de amostragem e método de Bitterlich.

\section{COMPARISON OF SAMPLING METHODS FOR STRUCTURAL ANALYSIS OF UNEVEN-AGED STANDS}

\begin{abstract}
This work was carried out in a uneven-aged stand at the Universidade Federal de Viçosa, Minas Gerais, Brazil, to compare estimates of the number of trees per hectare, volume per hectare, basal area per hectare, medium diameter and medium height of the stand and number of species, using plots of equal size (Method I) and the horizontal point sampling (Bitterlich Method), with basal area factors $K=1, K=2$ and $K=4$ (Methods II, III and IV, respectively). After analyses, it was verified that: a) to meet to a certain sampling error, a larger number of sampling points is needed in comparison with the number of plots of equal size; b) no significant difference was found among the volume estimates per hectare, basal area per hectare, medium diameter and medium height of the stand, obtained from the plots of equal size and the sampling points, regardless of the basal area factor; c) the horizontal point sampling (Bitterlich Method) can be used for characterization of the number of species in an uneven-aged stand if there is an increase of number of points or if the forest presents low species diversity; d) there was no significant difference among the numbers of trees per hectare and diameter class for the sampling methods studied. Methods II, III and IV underestimate the number of trees in the largest DAP classes and overestimate it in the smallest ones.
\end{abstract}

Key words: $\quad$ Uneven-aged stand, sampling methods and Bitterlich method.

1 Recebido para publicação em 8.5.2001.

Aceito para publicação em 6.11.2002.

2 Eng. Florestal, M.S., INPACEL AGROFLORESTAL LTDA - DR 001 Km 07 - 84990-000 Arapoti-PR, ${ }^{3}$ Prof. do Departamento de Engenharia Florestal da UFV, DEF/UFV, 36571-000 Viçosa-MG. 


\section{INTRODUÇÃO}

Os produtos florestais são utilizados em diversas atividades, desde as construções civis aos adornos de madeira, cobrindo uma variada gama de utilidades, indispensáveis ao bem-estar do homem.

Dentro desse contexto surge como alternativa o manejo florestal, cujo princípio é a manutenção da produção de bens e serviços em quantidade e qualidade para gerações presentes e futuras. Para isto, estimativas sobre características das populações florestais devem ser obtidas através de procedimentos de inventário florestal, definidos em função dos recursos disponíveis, da precisão requerida e dos objetivos do inventário.

Além dos planos de manejo, em várias situações há a necessidade de realização de inventários florestais, como partilha de bens, autuação perante crime ambiental, avaliação de propriedades para a venda, entre outros.

Em virtude das limitações de recursos financeiros, tempo, mão-de-obra, acesso e tamanho das florestas, é impraticável inventariar $100 \%$ da área das florestas. Assim, há a necessidade do emprego de métodos de amostragem, com o objetivo de obter estimativas precisas e eficientes de diferentes parâmetros populacionais de interesse.

Tradicionalmente, o método de amostragem mais utilizado para inventariar florestas eqüiâneas e ineqüiâneas é o método que se baseia na alocação de parcelas de área fixa. Este método geralmente possui custo elevado e exige maior tempo para os levantamentos, devido à marcação e medição de um grande número de árvores (Husch et al., 1982). Assim sendo, torna-se importante analisar diferentes métodos de amostragem, visando reduzir o tempo e o custo de execução do inventário, sem perda de precisão.

Considerando os métodos de amostragem alternativos, com o objetivo de reduzir os custos dos inventários florestais, sem, contudo, reduzir a sua precisão, tem-se o método idealizado por Bitterlich, em 1948. Devido à simplicidade do procedimento para obtenção dos dados, a aplicação desse método pode ser de extrema utilidade, principalmente em situações em que se necessita de um diagnóstico rápido do estoque de madeira entre outras características de florestas ineqüiâneas, que pela sua natureza demandam maiores esforços de amostragem. Diante deste fato, verifica-se a necessidade de comparar o método de amostragem que se baseia na alocação de parcelas de área fixa (método tradicional) com a amostragem por ponto horizontal (método de Bitterlich), em um inventário de floresta ineqüiânea, baseando-se em parâmetros como o número de árvores por hectare, o volume por hectare, a área basal por hectare, o número de espécies, o diâmetro médio e a altura média do povoamento, bem como na distribuição diamétrica, caracterizada pela distribuição do número de árvores em classes diamétricas.

\section{MATERIAL E MÉTODOS}

\subsection{Caracterização da Área}

O presente trabalho foi realizado em uma área localizada no município de Viçosa, Zona da Mata de Minas Gerais (Lat. 42 $53^{\prime} \mathrm{W}$ e Long. $20^{\circ} 45^{\prime} \mathrm{S}$ ). A área de estudo é conhecida como Mata da Praça de Esportes, possui 10,65 ha e é de propriedade da Universidade Federal de Viçosa.

As formações florestais existentes na região são caracterizadas como floresta estacional semidecidual, nos estádios primário e secundário, sujeitas a um ritmo estacional, com 20 a 50\% de árvores caducifólias na estação seca. Seus agrupamentos remanescentes mais expressivos localizam-se nas encostas superiores dos terrenos da região (Coelho, 1999).

Os dados para realização deste trabalho foram coletados mediante inventário florestal, utilizando os seguintes métodos de amostragem: método I (parcelas de área fixa - método-padrão), método II (Bitterlich, $\mathrm{K}=1$ ), método III (Bitterlich, $\mathrm{K}=2$ ) e método IV (Bitterlich, $\mathrm{K}=4$ ).

Para o método de amostragem que utiliza parcelas de áreas fixas, foram lançadas, sistematicamente, 13 parcelas ao longo de toda a área, dispostas no sentido norte - sul, distantes $90 \mathrm{~m}$ umas das outras. As parcelas possuíam $20 \times 25 \mathrm{~m}\left(500 \mathrm{~m}^{2}\right)$, sendo a maior dimensão alocada no sentido sul, na maior declividade. Para o método de Bitterlich, foi empregada a amostragem sistemática que utiliza o relascópio de Bitterlich. O centro de cada parcela de área fixa foi considerado como um ponto de amostragem, totalizando 13 pontos de amostragem. O nível de inclusão adotado foi de $5 \mathrm{~cm}$ de DAP (diâmetro à altura do peito). Em cada árvore amostrada foram feitas medições de CAP (circunferência à altura do peito), estimação de altura comercial e altura total e identificação de material botânico. 
As estimativas dos parâmetros populacionais área basal por hectare, número de árvores por hectare, volume por hectare, diâmetro médio, altura média e número de espécies para cada parcela e para cada ponto foram obtidas com as expressões apresentadas no Quadro 1.

Considerando o estádio de sucessão da Mata da Praça de Esportes, o volume com casca de cada árvore individual foi obtido pela equação (FUNDAÇÃO ...CETEC, 1995):

$$
\hat{V} i=0,00007423 . \text { dap }^{1,707348} . H t^{1,16873} ; \quad \mathrm{R}^{2}=97,30 \%
$$

em que $d a p=$ diâmetro à altura do peito, em $\mathrm{cm} ; H t=$ altura total, em $\mathrm{m}$.

\subsection{Cálculo do Tamanho da Amostra (n) e Erro de amostragem (E\%)}

Utilizando os volumes por hectare das parcelas e dos pontos de amostragem (Bitterlich), calculou-se o tamanho da amostra, para os quatro métodos de amostragem, através das seguintes expressões (Loetsch \& Haller, 1964):

\begin{tabular}{|c|c|}
\hline Parcelas de Áreas Fixas & Bitterlich \\
\hline$n=\frac{t^{2} C V^{2}}{E \%^{2}+\frac{t^{2} C V^{2}}{N}}$ & $n=\frac{t^{2} C V^{2}}{E \% \%^{2}}$ \\
\hline
\end{tabular}

em que $n=$ tamanho da amostra; $t=$ valor tabelado da distribuição t de Student, $(\alpha \%, \mathrm{n}-1 \mathrm{gl}) ; C V=$ coeficiente de variação; $E \%=$ erro de amostragem admissível; e $N=$ número total de unidades da população.

Neste trabalho foram adotados um erro de amostragem admissível (E\%) de $20 \%$ e o nível de probabilidade de $90 \%$, conforme a Portaria $n^{\circ} 054$ do IEF, de 25 de agosto de 1997.

O erro de amostragem E (\%) foi obtido pela seguinte expressão, para os quatro métodos de amostragem (Loetsch \& Haller, 1964):

Quadro 1 - Estimadores dos parâmetros populacionais para parcelas de área fixa (método I) e para amostragem por pontos horizontais (métodos II, III e IV)

Table 1 -Estimators of the populational parameters for plots of equal size (method I) and horizontal point sampling (methods II, III and IV)

\begin{tabular}{|l|c|c|}
\hline & Método I & Métodos II, III e IV \\
\hline Área basal por hectare & $B=\sum_{i=1}^{n} \frac{A s_{i}}{A}$ & $B=n^{*} K$ \\
\hline Número de árvores por hectare & $N / h a=\frac{n}{A}$ & $N / h a=\sum_{i=1}^{n} \frac{K}{A s_{i}}=\sum_{i=1}^{n} N_{i}$ \\
\hline Volume por hectare & $V / h a=\sum_{i=1}^{n} \frac{V i}{A}$ & $V / h a=\sum_{i=1}^{n} V i * N_{i}$ \\
\hline Diâmetro médio & $q=\sqrt{\frac{\sum_{i=1}^{n} d_{i}{ }^{2}}{n}}$ & $q=\sqrt{\frac{\sum_{i=1}^{n} d_{i}{ }^{2} * N_{i}}{\sum_{i} N_{i}}}$ \\
\hline Altura média & Somatório do número de espécies encontradas em cada parcela ou ponto \\
\hline Número de espécies & $\bar{H}=\frac{\sum_{i=1}^{n} H_{i}}{n}$ \\
\hline
\end{tabular}

em que $A s_{i}=$ área seccional da i-ésima árvore amostrada na parcela ou qualificada no ponto de amostragem, em $\mathrm{m}^{2} ; A=$ área da parcela em ha; $n=$ número de árvores amostradas na parcela ou qualificadas no ponto de amostragem; $V i=$ volume da i-ésima árvore amostrada, $\mathrm{em} \mathrm{m}^{3} ; d i=$ diâmetro da i-ésima árvore amostrada, em $\mathrm{cm} ; K=$ fator de área basal, em $\mathrm{m}^{2} / \mathrm{ha} ; N i=$ número de árvores por hectare que a i-ésima árvore qualificada representa; e $H i=$ altura da i-ésima árvore. 


$$
E \%=\frac{ \pm S_{\bar{x}} * t}{\bar{x}} * 100
$$

em que $S_{\bar{x}}=$ erro-padrão da média; $t=$ valor tabelado de $\mathrm{t}(10 \%$ e n-1 gl); e $\bar{x}=$ média dos volumes por hectare das parcelas e dos pontos de amostragem.

\subsection{Comparação Entre os Métodos de Amostragem}

A comparação entre os métodos de amostragem, considerando o parâmetro volume por hectare, foi realizada mediante análise do coeficiente de correlação e do teste F de Graybill (1976).

\subsubsection{Coeficiente de Correlação}

O coeficiente de correlação é definido como o grau de associação entre duas variáveis aleatórias, ou como a estimativa da medida da variação (em conjunto) de duas séries de variáveis. É um valor adimensional que dá uma idéia razoável da dependência apresentada por duas séries de variáveis. Os valores de uma série podem ser de correlação negativa ou positiva, ou ainda podem não estar associados (Graner, 1996).

Para o cálculo da correlação entre as estimativas de cada parâmetro, utilizou-se a expressão:

$$
r_{x y}=\frac{\operatorname{Cov}(x, y)}{s_{x} s_{y}}
$$

em que $x=$ método-padrão (parcelas de área fixa); $y=$ métodos alternativos (Bitterlich, $\mathrm{K}=1,2$ e 4); $\operatorname{Cov}(x, y)=$ covariância de $x$ em relação a $y ; S_{x}=$ desvio-padrão de $x$; e $S_{y}=$ desvio-padrão de $y$.

\subsubsection{Teste F de Graybill $\left(\mathrm{H}_{0}\right)$}

O teste $\mathrm{F}$ de Graybill, além de ter sido utilizado na comparação entre as estimativas de diferentes parâmetros populacionais obtidos nas parcelas e nos pontos, também foi na comparação da distribuição diamétrica obtida pelos quatro métodos de amostragem.

Foram ajustados modelos de regressão que envolvem o método-padrão (parcelas de área fixa - método I) e os métodos alternativos (Bitterlich - métodos II, III e IV, respectivamente):

$$
Y=\beta_{0}+\beta_{1} X+\varepsilon
$$

R. Árvore, Viçosa-MG, v.26, n.5, p.541-548, 2002 em que $Y=$ variável no método alternativo; $\beta_{0}$ e $\beta_{1}=$ parâmetros do modelo; $X=$ variável no método-padrão; e $\varepsilon=$ erro aleatório.

A similaridade entre as variáveis, considerando os quatro métodos de amostragem, foi verificada ao testar a hipótese simultânea:

$H_{0}:\left[\begin{array}{l}\beta_{0} \\ \beta_{1}\end{array}\right]=\left[\begin{array}{l}0 \\ 1\end{array}\right]$

$H a$ : complemento de $H_{0}$

Para testar essa hipótese aplicou-se a estatística F, conforme proposto por Graybill (1976):

$$
F\left(H_{0}\right)=\frac{(\widehat{\beta}-\theta)^{\prime}\left(X^{\prime} X\right)^{-1}(\widehat{\beta}-\theta)}{2^{*} Q M \operatorname{Re} s}
$$

em que

$\widehat{\beta}=\left[\begin{array}{c}\hat{\beta}_{0} \\ \hat{\beta}_{1}\end{array}\right]=$ vetor de coeficientes da equação de regressão;

$\theta=\left[\begin{array}{l}0 \\ 1\end{array}\right] ;\left(X^{\prime} X\right)^{-1}=\left[\begin{array}{cc}n & \sum x \\ \sum x & \sum x^{2}\end{array}\right]^{-1}$

QMRes = quadrado médio do resíduo; e

$F_{t a b}=[5 \% ; 2, \mathrm{n}-2 \mathrm{gl}]$.

\section{RESULTADOS E DISCUSSÕES}

\subsection{Erro de Amostragem e Tamanho da Amostra}

Analisando as estatísticas apresentadas no Quadro 2, verifica-se que as estimativas das médias e dos desviospadrão dos volumes por hectare são maiores para os métodos II, III e IV (Bitterlich $-\mathrm{K}=1, \mathrm{~K}=2$ e $\mathrm{K}=4$ ), em comparação com o método I (parcelas de área fixa), para uma mesma intensidade amostral.

Assim sendo, verifica-se que o número de unidades amostrais foi suficiente para atender ao erro de amostragem admissível de $20 \%$, a $90 \%$ de probabilidade, de acordo com a Portaria $\mathrm{n}^{\circ}$ 054, de 25/08/97 do Instituto Estadual de Florestas (IEF), somente para o método I 
(parcelas de área fixa). Para os métodos II (Bitterlich, $\mathrm{K}=1$ ), III (Bitterlich, $\mathrm{K}=2$ ) e IV (Bitterlich, $\mathrm{K}=4$ ), o número de pontos amostrais não atende à precisão requerida, sendo necessário lançar 19 pontos amostrais para o método II, 31 para o método III e 41 para o método IV, isto é, aumentando-se o fator de área basal (K), aumenta-se o número de unidades amostrais para satisfazer à precisão requerida. Estes resultados são semelhantes ao encontrado por Couto et al. (1990) em plantios de Eucalyptus saligna. Com isso, verifica-se a necessidade de um número maior de pontos de amostragem para satisfazer a um determinado erro de amostragem, em comparação com o método de amostragem baseada em parcelas de área fixa.

Analisando os gráficos da Figura 1 constata-se que, exceto para o parâmetro número de espécies, não existe tendência de os métodos de amostragem (parcelas de áreas fixas e variáveis) sempre superestimarem ou subestimarem os parâmetros volume por hectare, área basal por hectare, número de árvores por hectare, diâmetro médio e altura média das árvores. Somente em alguns casos os métodos de amostragem com base no método

Quadro 2 - Volum es das parcelas e dos pontos de am ostragem, em m ${ }^{3}$ ha; erro de am ostragem adm issível (E\%), em porcentagem; m édia dos volum es $(\bar{x})$; desvioppadrão $\left(S_{x}\right)$, em $\mathrm{m}^{3}$ hha; erro-padrão $\left(S_{\bar{x}}\right)$, em $\mathrm{m}^{3}$ ha, coeficiente de variação (CV), em porcentagem; área das parcelas (Á rea), em hectare; núm ero total de unidades am ostrais (N); tam anho da am ostra (n) e erro de am ostragem observado, em porcentagem

Table 2 -Volum es of plots and sampling points, in $\mathrm{m}^{3} / \mathrm{ha}$; acceptable sam pling error ( $\Theta \circ$ ), in percentage; volum e average $(\bar{x})$; standard deviation $\left(S_{x}\right)$, in $\mathrm{m} 3 / \mathrm{ha}$; standard error $\left(S_{\bar{x}}\right)$, in $\mathrm{m} 3 / \mathrm{ha}$; coefficient of variation $(C V)$, in percentage; $p l o t$ area (A rea), in hectare; total num ber of sam pling un its $(N)$; sam ple size (n) and observed sam pling error, in percentage

\begin{tabular}{|c|c|c|c|c|}
\hline \multirow{2}{*}{ Parcela } & \multicolumn{4}{|c|}{ Volume $\left(\mathrm{m}^{3} / \mathrm{ha}\right)$} \\
\hline & Método I & Método II & Método III & Método IV \\
\hline 1 & 28,57 & 30,97 & 30,09 & 12,79 \\
\hline 2 & 87,77 & 102,27 & 75,79 & 90,85 \\
\hline 3 & 77,46 & 93,13 & 106,33 & 83,59 \\
\hline 4 & 51,50 & 51,63 & 47,63 & 62,32 \\
\hline 5 & 86,69 & 92,29 & 90,44 & 122,42 \\
\hline 6 & 68,62 & 88,78 & 89,69 & 80,20 \\
\hline 7 & 36,29 & 17,08 & 13,10 & 26,20 \\
\hline 8 & 101,80 & 109,38 & 152,26 & 213,64 \\
\hline 9 & 63,37 & 63,23 & 92,94 & 93,52 \\
\hline 10 & 108,27 & 42,51 & 28,37 & 17,24 \\
\hline 11 & 71,62 & 84,79 & 69,09 & 22,92 \\
\hline 12 & 109,07 & 154,12 & 175,47 & 205,94 \\
\hline 13 & 134,92 & 142,13 & 185,56 & 191,92 \\
\hline E\% & 20 & 20 & 20 & 20 \\
\hline $\bar{x}$ & 78,92 & 82,49 & 88,98 & 94,12 \\
\hline$S_{x}$ & 30,38 & 40,73 & 55,00 & 71,11 \\
\hline$S_{\bar{x}}$ & 8,16 & 11,29 & 15,25 & 19,72 \\
\hline$C V(\%)$ & 38,49 & 49,37 & 61,81 & 75,55 \\
\hline ÁREA & 0,05 & & & \\
\hline $\mathrm{N}$ & 213,00 & & & \\
\hline$n$ & 12 & 19 & 31 & 41 \\
\hline Erro amostragem (\%) & 18,41 & 24,38 & 30,52 & 37,30 \\
\hline
\end{tabular}


de Bitterlich (métodos II, III e IV) tendem a superestimar os parâmetros avaliados, o que se deve principalmente ao número de árvores amostradas pelas parcelas de áreas fixas (81 árvores em média) e ao número de árvores qualificadas nos pontos de amostragem (em média, 14 árvores no método II, 7 árvores no método III e 4 árvores no método IV). Myers \& Beers (1971), comparando amostragem por pontos e amostragem por parcelas de área fixa, usando fator de área basal 10 (pés quadrados por acre) e parcelas de aproximadamente $800 \mathrm{~m}^{2}$, constataram que, em termos de número de árvores, a amostragem por pontos selecionou quase a metade do número de árvores selecionadas pela amostragem com parcelas de área fixa. Neste estudo, a proporção foi $17,28 \%$ no método II (Bitterlich, $\mathrm{K}=1$ ), 8,64\% no método III (Bitterlich,
$\mathrm{K}=2$ ) e 4,94\% no método IV (Bitterlich, $\mathrm{K}=4$ ).

A grande diferença entre o número de espécies amostradas pelos quatro métodos, (o método I - 108 espécies e os métodos II, III e IV - 54, 38 e 27 espécies, respectivamente), se deve ao número de árvores amostradas em cada método. Esta diferença permite inferir sobre a necessidade de um número maior de pontos de amostragem para caracterização de composição florística, em florestas com alta diversidade de espécies, ou uma melhor eficiência da amostragem por pontos para caracterização da composição florística em florestas com baixa diversidade de espécies, ou em povoamentos "puros" de espécies como, por exemplo, quaresminha (Miconia cinnamomifolia Nudir) e candeia (Vanylosmopsis erythropappa Sch. Bip.).
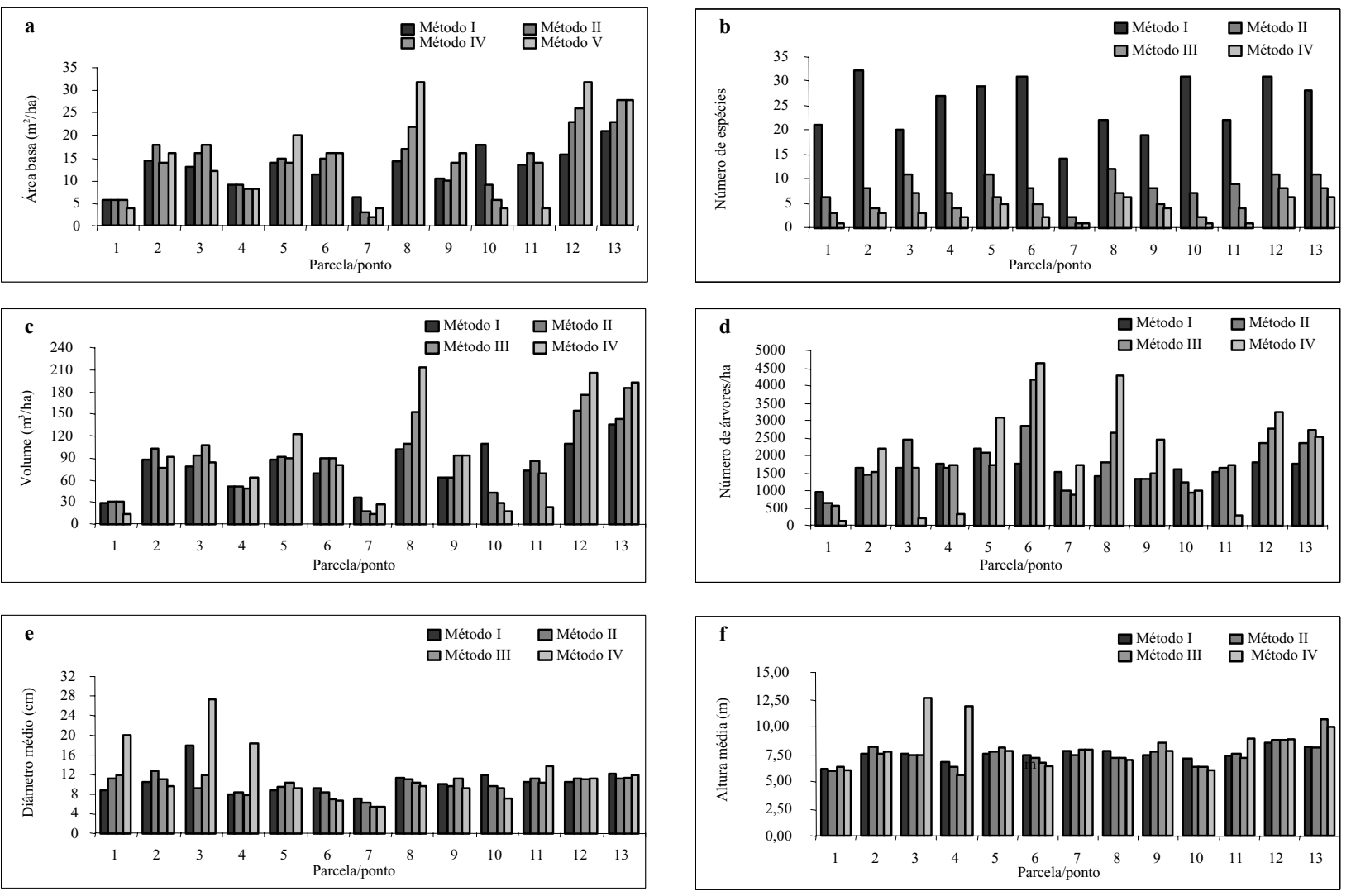

Figura 1 - Estimativas de área basal por hectare (a), número de espécies (b), volume por hectare (c), número de árvores por hectare (d), diâmetro médio (e) e altura média (f) das árvores, obtidas pelo método I (parcelas de áreas fixas) e pelos métodos II, III e IV (Bitterlich - $\mathrm{K}=1, \mathrm{~K}=2, \mathrm{~K}=4$ ).

Figure 1 -Estimates of basal area per hectare (a), number of species (b), volume per hectare (c), number of stems per hectare (d), mean diameter (e) and mean height (f) of the trees, obtained by method I (plots of equal size) and by methods II, III and IV (Bitterlich $-K=1, K=2, K=4$ ). 


\subsection{Comparação entre os Métodos de Amostragem}

As estimativas dos coeficientes de correlação e dos testes F de Graybill, para os parâmetros área basal por hectare, volume por hectare, número de árvores por hectare, número de espécies, diâmetro médio e altura média, envolvendo o método I (parcelas de áreas fixas) e os métodos II, III e IV (Bitterlich $-\mathrm{K}=1, \mathrm{~K}=2 \mathrm{e}$ $\mathrm{K}=4$ ), estão apresentadas no Quadro 3.

Quadro 3 - Estimativas dos coeficientes de correlação e testes F de Graybill (FHo) para os parâmetros área basal por hectare, volume por hectare, número de árvores por hectare, número de espécies, diâmetro médio e altura média do povoamento, envolvendo o método I (parcelas de áreas fixas) e os métodos II, III e IV (Bitterlich $-\mathrm{K}=1, \mathrm{~K}=2$ e $\mathrm{K}=4$ )

Table 3 - Estimates of the coefficients of correlation and tests F of Graybill (FHo) for the parameters basal area per hectare, volume per hectare, number of stems per hectare, number of species, mean diameter and mean height of stand, involving the method I (plots of equal size) and the methods II, III and IV (Bitterlich-K $=1$, $K=2, K=4$ )

\begin{tabular}{|l|l|c|c|}
\hline Variável & Método & Correlação & $\mathrm{F}\left(\mathrm{H}_{0}\right)$ \\
\hline \multirow{3}{*}{ Área basal por hectare } & I x II & 0,7723 & $0,4223 \mathrm{~ns}$ \\
\cline { 2 - 4 } & I x III & 0,6843 & $0,7490 \mathrm{~ns}$ \\
\cline { 2 - 4 } & I x IV & 0,5455 & $0,5075 \mathrm{~ns}$ \\
\hline \multirow{3}{*}{ Volume por hectare } & I x II & 0,7826 & $0,1371 \mathrm{~ns}$ \\
\cline { 2 - 4 } & I x III & 0,7461 & $0,9158 \mathrm{~ns}$ \\
\cline { 2 - 4 } & I x IV & 0,6968 & $1,3064 \mathrm{~ns}$ \\
\hline \multirow{2}{*}{$\begin{array}{l}\text { Número de árvores por } \\
\text { hectare }\end{array}$} & I x II & 0,6781 & $1,11 \mathrm{~ns}$ \\
\cline { 2 - 4 } & I x III & 0,4301 & $0,64 \mathrm{~ns}$ \\
\cline { 2 - 4 } & I x IV & 0,3381 & $0,56 \mathrm{~ns}$ \\
\hline \multirow{3}{*}{ Número de espécies } & I x II & 0,3861 & $276,231^{*}$ \\
\cline { 2 - 4 } & I x III & 0,2804 & $571,479^{*}$ \\
\cline { 2 - 4 } & I x IV & 0,2526 & $783,307^{*}$ \\
\hline \multirow{3}{*}{ Diâmetro médio } & I x II & 0,6869 & $0,3307 \mathrm{~ns}$ \\
\cline { 2 - 4 } & I x III & 0,6037 & $0,2000 \mathrm{~ns}$ \\
\cline { 2 - 4 } & I x IV & 0,6019 & $1,2964 \mathrm{~ns}$ \\
\hline \multirow{3}{*}{ Altura média } & I x II & 0,8772 & $0,0135 \mathrm{~ns}$ \\
\cline { 2 - 4 } & I x III & 0,7584 & $0,2835 \mathrm{~ns}$ \\
\cline { 2 - 4 } & I x IV & 0,21153 & $1,2112 \mathrm{~ns}$ \\
\hline
\end{tabular}

$\mathrm{ns}=$ não-siginificativo a $95 \%$ de probabilidade $\mathrm{e}^{*}=$ significativo a $95 \%$ de probabilidade.

Analisando os coeficientes de correlação no Quadro 3, verifica-se que existe maior grau de associação entre os métodos I e II (parcelas de áreas fixas e Bitterlich $-\mathrm{K}=1$ ), considerando todos os parâmetros avaliados. À medida que o fator de área basal aumenta $(\mathrm{K}=2$ e $\mathrm{K}=4)$ a correlação diminui, ou seja, existe menor correlação entre o método I e os métodos III e IV. Os resultados dos testes $\mathrm{F}$ de Graybill mostram que não existe diferença significativa entre os métodos de amostragem para os diversos parâmetros avaliados, exceto para o parâmetro número de espécies. Esta diferença se deve à discrepância entre o número de árvores amostradas nos métodos II, III e IV, conforme discutido anteriormente.

Diante dos resultados apresentados no Quadro 3, verifica-se que o método idealizado por Bitterlich em 1948 atende aos objetivos de diagnóstico rápido de florestas, uma vez que estima parâmetros importantes para quantificação dos recursos madeireiros com igual precisão ao método de parcelas de área fixa, para um mesmo número de unidades amostrais. Além disto, a existência de diferença estatística entre o método que utiliza parcelas de área fixa e os métodos de amostragem que utilizam o método de Bitterlich, para o parâmetro número de espécies, para mesma intensidade amostral, permite inferir que o método idealizado por Bitterlich poderá ser empregado em estudos de composição florística somente se houver aumento da intensidade amostral.

\subsection{Análise da Distribuição Diamétrica}

A comparação entre os números de árvores por hectare e por classe de diâmetro, obtidos pelos métodos de amostragem, foi realizada também com o teste $F$ de Graybill. Os resultados dos testes para a variável número de árvores por hectare e por centro de classe de DAP, para os métodos I (parcelas de áreas fixas) e II (Bitterlich $-\mathrm{K}=1$ ); I (parcelas de áreas fixas) e III (Bitterlich $-\mathrm{K}$ $=2$ ); e I (parcelas de áreas fixas) e IV (Bitterlich $-\mathrm{K}=$ 4), foram 11,28, 120,81 e 166,95, respectivamente, sendo todos significativos a $95 \%$ de probabilidade. Ao contrário do parâmetro número de árvores por hectare, os resultados dos testes F de Graybill indicam que existe diferença estatística entre o número de árvores por hectare por classe de DAP, entre os método que utiliza parcelas de área fixa e o método de Bitterlich, com fatores de área basal $\mathrm{K}=1, \mathrm{~K}=2 \mathrm{e} \mathrm{K}=4$. Além disto, as diferenças entre os números de árvores por hectare e por classe de DAP, apresentadas no Quadro 4, mostram que os métodos II, III e IV superestimam o número de árvores nas menores classes de DAP e subestimam-no nas maiores. Mesmo apresentando estas diferenças estatísticas, os quatro métodos de amostragem estudados caracterizaram a estrutura diamétrica como J - invertido, isto é, conforme o padrão de distribuição diamétrica das florestas ineqüiâneas. 
Quadro 4 - Número de árvores por hectare e por classe de diâmetro, para o método I (parcelas de áreas fixas) e os métodos II, III e IV (Bitterlich $-\mathrm{K}=1, \mathrm{~K}=2$ e $\mathrm{K}=4$ ) e estimativas das diferenças entre eles

Table 4 - Number of stems per hectare and per diameter class, for method I (plots of equal size) and methods II, III and IV (Bitterlich $-K=1, K=2$ and $K=4$ ) and estimates of the differences among them

\begin{tabular}{|c|r|r|r|r|r|r|r|}
\hline \multirow{2}{*}{ Centro de Classe (cm) } & \multicolumn{4}{|c|}{ Número de Árvores por Hectare } & \multicolumn{3}{|c|}{ Diferença do Número de Árvores } \\
\cline { 2 - 8 } & \multicolumn{1}{|c|}{ I } & \multicolumn{1}{|c|}{ II } & \multicolumn{1}{|c|}{ III } & \multicolumn{1}{|c|}{ IV } & \multicolumn{1}{c|}{ (II-I) } & \multicolumn{1}{|c|}{ (III-I) } & (IV-I) \\
\hline 7,5 & $1.210,40$ & $1.282,55$ & $1.433,08$ & $1.549,89$ & 72,14 & 222,67 & 339,48 \\
12,5 & 243,00 & 296,89 & 250,03 & 279,64 & 53,88 & 7,02 & 36,63 \\
17,5 & 92,28 & 127,96 & 119,77 & 78,20 & 35,68 & 27,48 & $-14,07$ \\
22,5 & 43,06 & 39,55 & 51,52 & 71,31 & $-3,51$ & 8,45 & 28,24 \\
27,5 & 16,91 & 11,97 & 15,93 & 20,60 & $-4,94$ & $-0,99$ & 3,68 \\
32,5 & 1,53 & 0 & 0,00 & 0 & $-1,53$ & $-1,53$ & $-1,53$ \\
37,5 & 3,07 & 0,76 & 1,53 & 0 & $-2,31$ & $-1,54$ & $-3,07$ \\
42,5 & 0 & 0,59 & 1,18 & 0 & 0,58 & 1,17 & 0 \\
47,5 & 1,53 & 0,43 & 0,86 & 0 & $-1,10$ & $-0,67$ & $-1,53$ \\
\hline Total & $1.611,82$ & $1.760,71$ & $1.873,90$ & $1.999,66$ & 148,88 & 262,06 & 387,83 \\
\hline
\end{tabular}

\section{CONCLUSÕES E RECOMENDAÇÕES}

Após o desenvolvimento do trabalho, chegou-se às seguintes conclusões:

- Há a necessidade de número maior de pontos de amostragem para atender ao erro de amostragem estipulado em comparação com o método de parcelas de áreas fixas. Quanto maior o fator de área basal (K), maior será este número de pontos de amostragem.

- Há a necessidade de número maior de pontos de amostragem para caracterizar a composição florística de uma floresta em estádio de sucessão secundário na amostragem por ponto, em comparação com o método de parcela de área fixa, em virtude do menor número de árvores amostradas.

- O método de amostragem por pontos pode ser utilizado em inventários florestais diagnósticos, haja vista que parâmetros importantes para quantificação dos recursos florestais madeireiros foram estimados da mesma forma que aqueles estimados pelo método de área fixa.

\section{REFERÊNCIAS BIBLIOGRÁFICAS}

CHACKO, V. J. A manual on sampling techniques for forest surveys. New Delhi: Manager of Publications, 1964. $172 \mathrm{p}$.

R. Árvore, Viçosa-MG, v.26, n.5, p.541-548, 2002
COELHO, D. J. S. Modelo de gestão florestal sustentável para a microrregião de Viçosa, Minas Gerais. Viçosa-MG: Universidade Federal de Viçosa, 1999. 80 p. Dissertação (Mestrado em Ciência Florestal) - Universidade Federal de Viçosa, 1999).

COUTO, H.T.Z.; BASTOS, N.L.M.; LACERDA, J.S. A amostragem por pontos na estimativa da altura de árvores dominantes e número de árvores por hectare em povoamentos de Eucalyptus saligna. IPEF, n. 43/44, p. 50-53, 1990.

FUNDAÇÃO CENTRO TECNOLÓGICO DE MINAS GERAIS - CETEC - Determinação de equações volumétricas aplicáveis ao manejo sustentável de florestas nativas no estado de Minas Gerais e outras regiões do país. Belo Horizonte: 1995.295 p.

GRANER. E. A. Estatística. São Paulo: Escola Superior de Agricultura Luiz de Queiroz, 1966. 184 p.

GRAYBILL, F. A. Theory and application of the linear model. Massachusetts: Ouxburg Press, 1976. 704 p.

HUSCH, B.; MILLER, C.I.; BEERS, T. W. Forest mensuration. 3.ed. New York: John Wiley \& Sons, 1982. $402 \mathrm{p}$.

LOETSCH, F.; HALLER, K. E. Forest inventory. Munique: BLv Verlagsgesellschaft, 1964. v.1. 436 p.

MYERS, C. C.; BEERS, T. W. Point sampling compared for forest inventory. Department of Forestry and Conservation. Purdue: Purdue University, 1971 (Research Bulletin, 877). 